\title{
Investigation of the effects of Stewart platform-type industrial robot on stability of robotic milling
}

\author{
L. T. Tunc ${ }^{1} \cdot$ Jay Shaw ${ }^{1}$
}

Received: 10 October 2015 / Accepted: 25 January 2016 / Published online: 18 February 2016

(C) The Author(s) 2016. This article is published with open access at Springerlink.com

\begin{abstract}
Mobile machining with industrial robots is proposed as a cost-effective and portable manufacturing alternative to large scale $\mathrm{CNC}$ machine tools in large-scale part manufacturing. Robotic milling, one of the widely used mobile machining approaches, involves several technical challenges and distinct characteristics in terms of machining dynamics and stability due to completely different structural build up. In this paper, distinctive effects of Stewart platform-type of hexapod robot on stability of robotic milling is investigated based on characterisation of its structural dynamics, simulation of stability limits and experimental validation. Three aspects are demonstrated: (1) the positiondependent stability diagrams due to the position-dependent dynamics of the hexapod platform, (2) the effects of cross transfer function due to the complex kinematic chain on milling stability and (3) the role of feed rate direction in stability of robotic milling. The conditions for minimised positiondependent stability through appropriate tooling are also illustrated through simulations and experimental verification. The cases where process stability may be governed by either the hexapod robot or the cutting tool modes are discussed and identified through stability analysis. It is shown that the feed rate direction becomes a significant parameter for stability limits in robotic milling. The conditions at which the cross transfer function becomes significant on milling stability are discussed through simulations and experimental results. It is shown that cross transfer functions may significantly affect
\end{abstract}

L. T. Tunc

1.tunc@sheffield.ac.uk

1 Nuclear Advanced Manufacturing Research Centre, The University of Sheffield, Sheffield, UK milling stability especially when the radial depth of cut is less than $50 \%$ of the tool diameter. As one of the important outcomes of this research, it is found that appropriate tooling may decrease the reliance of milling stability on robot position.

Keywords Robotic milling $\cdot$ Stability $\cdot$ Machining dynamics

\section{Introduction}

Robotic milling is one of the widely proposed mobile machining approaches for manufacturing of large scale parts designed in aerospace, nuclear, automotive, and oil and gas industries, where high investment and operational costs are incurred due to use of the large scale CNC machine tools [1]. Within this perspective, robotic milling with industrial robots is considered to be a way to decrease costs through "process-to-part" approach offering increased flexibility and decreased lead time compared to CNC machine tools [1].

Robots were first used for handling, assembly and welding tasks in manufacturing industry. Recently, industrial robots have started to be used for mobile milling, i.e. robotic milling, and there are several proof-of-concept studies which identify challenges and provide solutions for challenges faced in robotic milling. Industrial robots are advantageous over $\mathrm{CNC}$ machine tools in terms of mobility, ease of installation and cost but they lack accuracy, robustness and stiffness for milling applications due to their flexible structures and simpler control system with respect to CNC machine tools. Thus, it is important to identify the associated challenges and the points where the requirements for metal cutting are not met in robotic milling. Chatter stability is one of the main limitations in machining operations, as well as in milling [2]. Therefore, it is important to investigate the challenges 
regarding process stability associated with robotic milling operations.

Robots are designed in serial or parallel configuration. In serial robots the link lengths are constant throughout the workspace, but in parallel robots, especially the ones actuated with extensible links, the link lengths vary together with the angular position throughout the robot motion. Even if the parallel kinematic configuration is not formed by extensible links, the point of contact between the link and the prismatic support may vary. In the literature, mostly serial robots have been studied to improve positional accuracy and dynamic stiffness for mobile machining. Gears and bearings are identified as the main sources of system compliance, which could be up to $50 \%$ or even $75 \%$ of the overall system compliance, depending on the size of the robot [3]. In one of the studies, Pan et al. [4] emphasised the differences between the CNC machine tools and a vertical articulated serial robot, where they identified chatter characteristics in robotic milling. Zaghbani et al. [5] studied the vibration response of robotic machining systems, where variable spindle speed strategy is applied. Vibration and cutting forces are used as the stability criterion.

Literature on dynamics of milling with parallel kinematic robots is scarce but there are noted studies on the use of parallel kinematics in machine tools as summarised in the rest of this section. The first use of parallel kinematic mechanisms in machining was in the form of triceps and hexapods as part of machine tools. The parallel kinematic mechanism was expected to improve the overall stiffness of the machine tools. It was also expected to provide better accuracy due to the motion in six degrees of freedom. In that perspective, the stiffness of hexapod machine tools are compared with the conventional CNC machine tools by Tlusty et al. [6] where several problems are addressed regarding the hexapod configuration. They mainly compared the use of serial and parallel kinematics for machine tools. They concluded that, contrary to expectations, the parallel kinematic mechanism (PKM) introduces additional flexibility for machine tool applications. The use of machine tools with PKM in productive industrial work is studied by Rehsteiner et al. [7]. They shared the results from users and manufacturers of such machine tools and suggested ways to improve the use of PKM in machining. Later, Fassi and Wiens [8] reviewed the use of PKM-type machine tools in machining industry. In this review paper, they mainly pointed out that the unfavourable transmission of motion hinders both positional accuracy and dynamic stiffness. In addition, the lack of comprehensive calibration techniques, weak or not well-defined dynamic behaviour, and complex control algorithms for compensation of stiffness variation among the workspace, are also pointed out. Weck and Staimer [9] reviewed the development of parallel kinematics for machine tools, and compared their application and performance to Cartesian machine tools based on the results from CIRP laboratories, machine tool manufacturers and users. It is concluded that further research and development is required to enable wide use of PKM-type machine tools. In one of the recent studies, the stiffness of machine tools in different configurations such as serial, parallel and hybrid are evaluated by Shneor and Portman [10]. They proposed a new stiffness-related engineering index called minimum stiffness value, which embraces both local and global stiffness over the workspace. Law et al. [11] studied position-dependent dynamics and stability of serialparallel scissor kinematic machine tools through a multibody dynamic machine model. They modelled the dynamics of the strut links using Timoshenko beam elements through substructuring based on improved reduced-order models. They compared the performance of full order FEM models and the reduced-order model through experimental results. Then, the position dependency in the dynamics of the machine tool is estimated for simulation of position-dependent stability maps. The experimental results showed that the model can estimate the amplitude of the dynamic response at the tool tip at a reasonable accuracy; however, slight discrepancies are observed in estimation of the natural frequency, which is due to several simplifications at the joints. In a recent study, a free-leg hexapod approach is proposed by Axinte et al. [12] for use of hexapod robots in mobile machining. In the proposed study, each leg can be individually positioned on the workpiece surface where they focused on development, calibration and exploitation of the proposed design. Their study showed that the positioning error could be decreased down to $50 \mu \mathrm{m}$.

Although the stiffness, chatter mechanism and applicability of industrial robots in mobile milling operations are studied in several papers, the unique challenges associated with the stability of robotic milling, especially with hexapod robots, have not been in the focus. Henceforth, this paper aims to contribute to the understanding of distinctive cases in stability of robotic milling operations utilising Stewart platform-type of hexapod robots. The tool point frequency response function (FRF) is measured through impact hammer tests to simulate stability diagrams based on the stability theory proposed by Budak and Altintas [13]. The cases where the hexapod robot may govern stability are identified through simulations and experimental results. The position-dependent stability limits associated with robotic milling are investigated through simulations and the findings are verified through experiments. It is shown that variable spindle strategy may be an effective way of dealing with position-dependent stability limits. The feed rate direction is identified to be an important parameter due to asymmetrical tool point FRF, which is caused by the effect of flexibility introduced by the hexapod robot. Cross transfer functions are generally ignored in milling stability analysis as they are not significant for $\mathrm{CNC}$ machine tools. However, it is found that the flexible hexapod structure amplifies the cross transfer function effect even at cutting tool modes. In this 
paper, the conditions where cross transfer function may be significant for stability are also studied through simulations and experimental results. Henceforth, the paper is organised as follows; the dynamics and stability of end milling is summarised in section 2 . This is followed by investigating the effects of the hexapod robot on milling stability in section 3 . The variable spindle speed strategy to deal with positiondependent stability is discussed in section 4 and the paper is finalised with conclusions and remarks in section 5.

\section{Dynamics and stability of end milling}

The dynamic flexibility caused by the cutting tool and/or workpiece results in dynamic vibrations, which causes modulated chip thickness and cutting forces. The flexible milling system becomes a self-excited regenerative system. The conditions where the vibrations grow, i.e. chatter, or diminish, i.e. stable, depend on the excitation frequency and system gain. In general, the excitation frequency is directly related to the spindle speed and the system gain is related to the system flexibility and cutting depth. Thus, it is important to establish the relation between the dynamic flexibility, cutting depth and the spindle speed. In this section, dynamics and stability of end milling is summarised based on the model proposed by Budak and Altintas [13].

\subsection{Dynamic cutting}

The cross section of a helical end mill, flexible in transverse directions $x$ and $y$, with $N$ number of cutting flutes is illustrated in Fig. 1. The equations of motion for this system in $x$ and $y$ directions are written as follows:

$m_{x} \ddot{x}+c_{x} \dot{x}+k_{x} x=F_{x}$

$m_{y} \ddot{y}+c_{y} \dot{y}+k_{y} y=F_{y}$

where $m, c$ and $k$ are the modal mass, damping and stiffness of the structure in each direction, respectively. The dynamic cutting forces acting on the tool, i.e. $F_{x}$ and $F_{y}$, depend on the dynamic chip thickness, $h_{j}$, axial depth of cut, $a$, radial depth of cut, $b$, and tool geometry. As the process stability depends on the dynamic vibrations at the tool tip, where cutting takes place, the above equation is applicable to robotic milling, as well. As long as milling stability is concerned it is always a function of the tool tip dynamics. Thus, regardless of the machining unit, i.e. a robot or a machine tool, behind the tool equations of motion at the tool tip can be represented by a combination of modes contributed by different structural components.

Each cutting edge removes modulated chip thickness as a wavy surface is generated in the previous cutting pass. The dynamic part of the chip thickness is written in terms

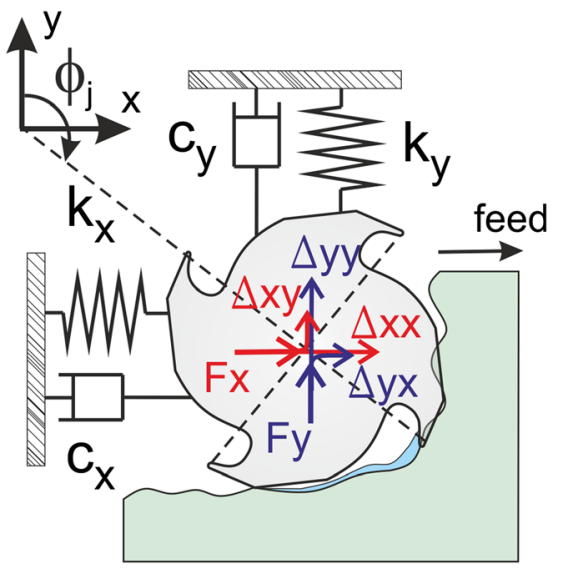

Fig. 1 Cross section of a flexible helical end mill

of the angular position, $\Phi_{j}$, of the $j^{\text {th }}$ cutting edge, and the dynamic displacements of the cutting tool in $x$ and $y$ directions as follows:

$h_{j}(\phi)=\left[\Delta x \sin \phi_{j}+\Delta y \cos \phi_{j}\right]$

The dynamic cutting forces are written in matrix form using the above definition [13]:

$\left\{\begin{array}{l}F_{x} \\ F_{y}\end{array}\right\}=\frac{1}{2} a K_{t}\left[\begin{array}{ll}a_{x x} & a_{x y} \\ a_{y x} & a_{y y}\end{array}\right]\left\{\begin{array}{c}\Delta x \\ \Delta y\end{array}\right\}$
where $\Delta x=\left(x_{c}-x_{c}^{o}\right)$ and $\Delta y=\left(y_{c}-y_{c}^{o}\right)$

In Eq. 3, $a_{x x}, a_{x y}, a_{y x}, a_{y y}$ stand for the directional coefficients which relate the dynamic displacements to the dynamic cutting forces [13], $K_{t}$ is the tangential cutting force coefficient, $\left(x_{c}, y_{c}\right)$ and $\left(x_{c}^{o}, y_{c}^{o}\right)$ are the inner and outer modulations, respectively.

In general, tool tip dynamics is governed by the direct and cross transfer functions contributed by different structural components in the machining unit. The direct transfer functions relate the excitation force to the vibration along the corresponding direction, whereas cross transfer functions (CTF) relate the excitation force to the vibration along the perpendicular direction to the excitation force. In other words, once a structure is excited in $-x$ direction, it is expected to vibrated both in $-x$ and $-y$ directions. The transfer functions relating the excitation force in $-x$ direction to the vibration in $-x$ and $-y$ directions are called to be Gxx and Gxy, respectively. These are graphically illustrated in Fig. 1, where Dxx and Dyx are the dynamic displacement in $-x$ direction excited by the dynamic force $F x$ and Dyy and Dxy are the dynamic displacement in $-\mathrm{y}$ direction excited by the dynamic force $F y$.

\subsection{Milling stability}

Equation (3) is rearranged after mathematical manipulations and equations of motion are written in frequency domain, 
where $\boldsymbol{A}_{\boldsymbol{0}}$ is the first order Fourier Transformation directional coefficient matrix of the milling system:

$$
\begin{aligned}
& \{F\} e^{i \omega_{c} t}=\frac{1}{2} a K_{t}\left(1-e^{-i \omega_{c} T}\right) \underbrace{\left[A_{0}\right]\left[G\left(i \omega_{c}\right)\right]}_{\left[G_{0}\left(i \omega_{c}\right)\right]}\{F\} e^{i \omega_{c} t} \\
& \text { where, }\left[G\left(i \omega_{c}\right)\right]=\left[\begin{array}{cc}
G_{x x}\left(i \omega_{c}\right) & G_{x y}\left(i_{c}\right) \\
G_{y x}\left(i \omega_{c}\right) & G_{y y}\left(i_{c}\right)
\end{array}\right]
\end{aligned}
$$

Finally, the stability of this system reduces to an eigenvalue problem considering that the above equation has a non-trivial solution only if its determinant is zero [13]:

$\operatorname{det}\left[[I]+\Lambda\left[G_{0}\left(i \omega_{c}\right)\right]\right]=0$

The eigenvalue, $\Lambda$, of Eq. (5) is written in terms of process parameters:

$\Lambda=-\frac{N}{4 \pi} K_{t} a\left(1-e^{-i \omega_{c} T}\right)$

Finally, the limiting stable cutting depth for the end milling system is written by expressing the eigenvalue, $\Lambda$, in terms of the transfer function of the system:

$a_{\lim }=-\frac{2 \pi \Lambda_{R}}{N K_{t}}\left(1+\kappa^{2}\right)$

where, $\Lambda=-\frac{1}{2 a_{0}}\left(a_{1} \pm \sqrt{a_{1}^{2}-4 a_{0}}\right)$

In Eq. (7), the eigenvalue $\Lambda$ is solved analytically to propose an analytical solution, where the cross transfer functions between perpendicular directions, i.e. $\mathrm{x}$ and $\mathrm{y}$, are ignored for cylindrical cutting tools. However, the eigenvalue needs to be solved numerically for the cases where the cross transfer function may be significant. $\Lambda_{R}$ is the real part of the eigenvalue $\Lambda . \kappa$ is the ratio between the imaginary and the real part of the eigenvalue, which is also written in terms of the chatter frequency, $\omega_{c}$, and tooth passing period, $T$. In the stability solution provided by Altintas and Budak [13], $a_{0}$ and $a_{l}$ are written in terms of the direct transfer functions of the system, $G_{x x}, G_{y y}$ and the average directional coefficients $\alpha_{x x}, \alpha_{y y}$, $\alpha_{x y}, \alpha_{y x}$, which are functions of the start and exit angles, and the radial cutting force coefficient.

\section{Effects of hexapod robot on milling stability}

There are several differences in robotic milling with a hexapod robot with respect to $\mathrm{CNC}$ machine tools and PKM type $\mathrm{CNC}$ machine tools in terms of structural build up, configuration, motion transmission and control system. Configuration of a hexapod $\mathrm{CNC}$ machine tool, a gantry type $\mathrm{CNC}$ machine tool and the robotic milling with hexapod robot studied in this paper are compared in Fig. 2.
In PKM type CNC machine tools (see Fig. 2a), the spindle is carried by six struts connected to the main frame of the machine tool. Both translation and rotation are provided by the linear and angular motion of these struts. The hexapod machine tools are typically configured in such a way that the spindle axis is along the gravitational direction and hence the struts are subjected to bending due to the weight of the spindle, tool holder and tool assembly. Consequently, the hexapod machine tool is flexible in all three directions, i.e. $x, y$ and $z$. Such a 3D flexibility is transferred to the tool coordinates by introduction of flexibility in all of the three directions of the cutting tool.

$\mathrm{CNC}$ machine tools are generally configured in axis on axis principal, where massive and rigid structural blocks carry the spindle. In 3-axis or 5-axis machine tools the translational and/ or rotary motion can be provided through several combinations of linear axes, i.e. $x, y, z$ and rotational axes, i.e. A, B, and C. Gantry type CNC machine tools (see Fig. 2b) are widely used in large-scale part manufacturing, where the $\mathrm{X}$ and $\mathrm{Z}$ axes are placed on the gantry $y$ axis and rotary motion is provided by the $\mathrm{A}$ and $\mathrm{C}$ axes on the milling head. Due to such a structural build up, gantry type $\mathrm{CNC}$ machine tools are generally flexible in $x$ and $y$ directions but rigid in the $z$ direction, i.e. spindle axis. Converting this to the tool coordinates, it can be said that the gantry type of machine tools are flexible in two transversal directions of the cutting tool but rigid in tool axis direction.

In robotic milling with a hexapod robot, i.e. Stewart platform, (see Fig. 2c) studied in this paper, the translational and rotational motion are provided by the six extensible struts similar to hexapod CNC machine tools, where in this case the struts are mounted to the ground. However, the spindle axis is horizontally aligned and hence the struts are not subjected to bending moment due to the weight of spindle, tool holder and tool assembly, which is instead in line with the axial direction of the struts. Thus, it can be said that such a hexapod setup is flexible in $x$ and $z$ directions but relatively more rigid in $y$ direction. Consequently, the hexapod setup introduces flexibility in the tool axis direction and one of the transversal directions of the cutting tool, where one of the transversal direction of the cutting tool is in line with the axis of the struts.

\subsection{Simulation of milling stability with the effect of hexapod robot}

The tool tip dynamics is affected by robot position due to the dynamic flexibility introduced by the hexapod robot [15]. Hence, milling stability in robotic milling will present typically different cases. In this section, the stability of robotic milling with the hexapod robot is investigated through simulations. 
Fig. 2 Comparison of $\mathrm{CNC}$ machine tool and the hexapod robot. (a) Hexapod type machine tool [14] (b) gantry type CNC machine tool $(\mathbf{c})$ robotic milling setup

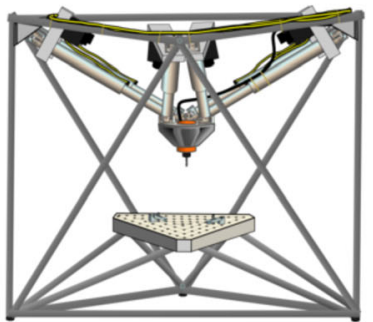

a

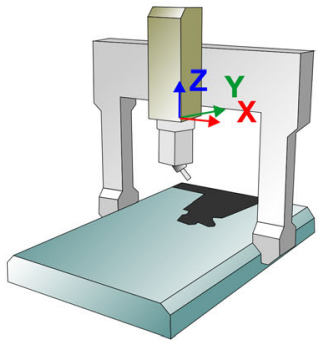

b

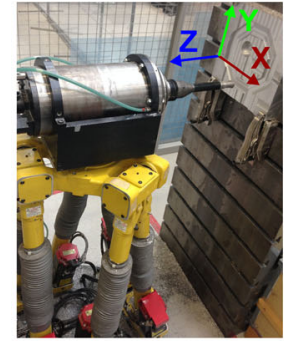

C

\subsubsection{Simulation of stability limits with the effect of hexapod robot}

The dynamically flexible structure of the hexapod robot may affect milling stability in various ways at different machining stages where robotic milling is employed, where several types of cutter may be used as represented in Table 1. Tool 1 has a long and flexible cutter body with relatively low natural frequency. tool 2 has relatively shorter body integrated to the tool holder with relatively high natural frequency. tool 3 represents a case where the tool is much stiffer than the hexapod as in face milling. The FRF measurements showed that the first natural frequency of tool 1 , tool 2 and tool 3 are 500, 2500, and $500 \mathrm{~Hz}$, respectively. The spindle introduces the first natural frequency around $100 \mathrm{~Hz}$ and the hexapod robot introduces compliance below $100 \mathrm{~Hz}$.

For a representative half-immersion down-milling case, the variation of chatter frequencies with spindle speed are simulated for the three cutting tools as plotted in Fig. 3. It is seen that the chatter frequency is around the natural frequency of tool 2 , which means that milling stability is dominated by the tool modes for tool 2. As tool 1 is considered, it is observed that the chatter frequency shifts down to $30 \mathrm{~Hz}$ at speeds around $5000 \mathrm{~Hz}$. Thus, it can be said that milling stability can be governed by the hexapod robot at a narrow band of spindle speed. In milling with tool 3, the chatter frequency changes between 30 and $120 \mathrm{~Hz}$, which are close to the natural frequency of the hexapod robot or spindle, respectively. So, it can be concluded that milling stability may be governed by the hexapod robot for operations such as face milling or end milling with long and slender tools.

\subsubsection{Effect of robot position on stability limits}

The spindle is carried by a moving platform, which is positioned and oriented by the motion of six struts and hence the position of the cutting tool is defined by the length and angle of these struts. Consequently, the stiffness and natural frequency of the hexapod robot becomes position dependant even for a simple 1D linear motion. The tool tip FRF may become position dependent if the dynamics of the hexapod robot affects the tool tip dynamics. For such cases the stability diagrams vary with the position of the robot. In other words, the modal parameters $\mathrm{G}\left(i \omega_{\mathrm{c}}\right)$ in Eq. (4) and hence the stability solution given in Eqs. (6) and (7) may vary with position of the robot depending on the interaction between the modes contributed by the tool and robot structures. The position dependency of stability diagrams is discussed for three cases

Table 1 Geometry of the tools used for comparison

\begin{tabular}{|c|c|c|c|c|c|}
\hline Tool No & Tool Type & Flutes & $\begin{array}{c}\text { Diameter } \\
(\mathrm{mm})\end{array}$ & $\begin{array}{l}\text { Length } \\
(\mathrm{mm})\end{array}$ & Photo \\
\hline Tool 1 & $\begin{array}{l}\text { Inserted } \\
\text { flat end mill }\end{array}$ & 2 & 25 & 160 & \\
\hline Tool 2 & $\begin{array}{l}\text { Inserted } \\
\text { flat end mill }\end{array}$ & 2 & 16 & 141 & \\
\hline Tool 3 & Face mill & 4 & 65 & 140 & \\
\hline
\end{tabular}




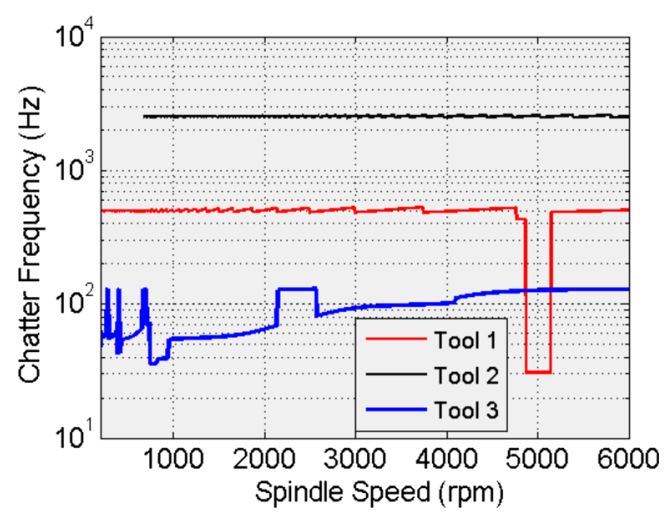

Fig. 3 Chatter frequencies for different tooling

through stability simulations, where the cases are listed in Table 2. The material is AISI316L stainless steel with cutting force coefficients $\mathrm{Ktc}=3200 \mathrm{MPa}$ and $\mathrm{Krc}=900 \mathrm{MPa}$.

The stability diagrams, simulated for representative random machining locations throughout the robot's workspace, are plotted in Fig. 4.

The variation of the stability diagrams with the machining location in case 1 is given in Fig. 4a. It is seen that the absolute stability limit is around $0.1 \mathrm{~mm}$ and the stable cutting depth reaches up to $0.3 \mathrm{~mm}$ at spindle speeds around $5000 \mathrm{rpm}$. The stability diagram shifts mainly in terms of spindle speed due change of natural frequency at the tool tip with machining location. It is also observed that the stability limits vary with the machining location. The stability diagrams simulated for case 2 are shown in Fig. 4b. The locations of the stability diagrams seem to be almost the same regardless of the machining location, except a slight change in the absolute stability limit. In Fig. 4c, the stability diagrams for case 3 are presented, which show major variations depending on the machining location. This is due to the fact that the robot modes are much dominant than the cutting tool. Especially at spindle speeds lower than $2000 \mathrm{rpm}$, the tooth passing frequency excites the robot modes with natural frequency varying between 20 and $100 \mathrm{~Hz}$ as observed in the frequency response functions.

In this section, it is shown that the machining location and tool type may significantly affect the stability diagrams due to the changing tool tip dynamics. Thus, it is important to find either the common stable regions among the machining locations or find the tooling conditions where the tool tip dynamics may not be affected by the robot position. Having

Table 2 Simulation cases for position dependant stability

\begin{tabular}{llll}
\hline Case & Tool & Milling mode & Radial depth of cut (\%) \\
\hline Case 1 & Tool 1 & Down & 40 \\
Case 2 & Tool 2 & Down & 30 \\
Case 3 & Tool 3 & Down & 50 \\
\hline
\end{tabular}

discussed the effect of machining location on stability of robotic milling, the next section discuss the effect of feed rate direction on stability of robotic milling.

\subsubsection{Effect of feed rate direction on stability of robotic milling}

The hexapod robot introduces asymmetric flexibility in $x$ and $y$ directions of the cutting tool. Thus, as a result of the interaction of the modes contributed by the robot and tool structure in $x$ and $y$ directions, the tool tip FRF becomes asymmetric in $x$ and $y$ directions of the cutting tool [15]. In other words, Gxx and Gyy in Eq. (4) becomes much different from each other, in terms of natural frequencies and amplitudes. In CNC machine tools, such an asymmetry is not as significant as in the robotic milling because the low frequency modes due to the machine tool structure are very rigid and hence their effects on tool tip dynamics are relatively very small or insignificant. In the stability solution [13], the feed direction is in $x$ direction and the cross feed direction is in $y$ direction. However, if the cutting feed rate direction is in any direction angled with respect to $x$, the transfer functions need to be oriented considering the feed rate direction [15]. For a representative case, the asymmetrical FRF introduced by the hexapod robot and the effect of the feed rate direction in end milling on oriented transfer functions and stability limits are demonstrated, through simulations, in Fig. 5. The FRFs measured at the tip of tool 1, where the tool is attached to $\mathrm{CNC}$ machine tool and to the hexapod robot are compared in Fig. 5a. It is seen that the FRF measured in $x$ and $y$ directions at tool 1 are asymmetric in terms of natural frequency, number of modes and amplitude when it is attached to hexapod robot. On the other hand, FRF in $x$ and $y$ directions are almost the same when tool 1 is attached to the CNC machine tool. This means that the transfer function oriented in the feed rate direction will significantly change in robotic milling as shown in Fig. 5b, however in CNC milling the oriented transfer function will not change with feed rate direction. As observed in Fig. 5b, the oriented transfer function in feed direction gradually diverges from Gxx to Gyy as the feed rate direction is changed from $0^{\circ}$ to $90^{\circ}$ with respect to $\mathrm{x}$ axis.

The effect of feed rate direction on stability diagrams is demonstrated for an end milling case with tool 1 , where the radial depth of cut is $60 \%$ and the material is AISI316L stainless steel. The stability diagrams are simulated for 5 feed rate directions as plotted in Fig. 5c. It is clearly seen that the stability lobes vary significantly in terms of spindle speed and stability limits as feed rate direction is varied from 0 to $90^{\circ}$. Such an occurrence causes the stable cutting conditions to vary throughout a machining cycle where the feed direction may change as in circular or rectangular toolpath strategies. Thus, it can be said that the machining strategy will significantly affect milling stability in robotic milling. 


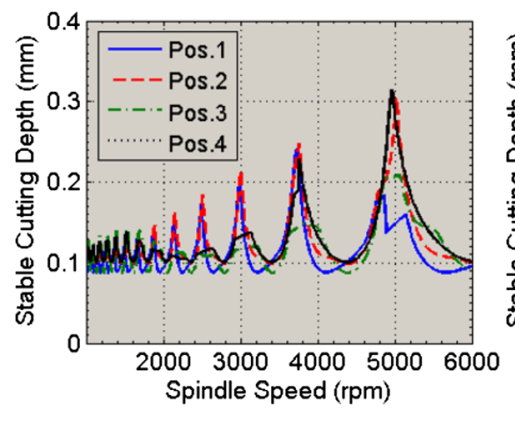

a

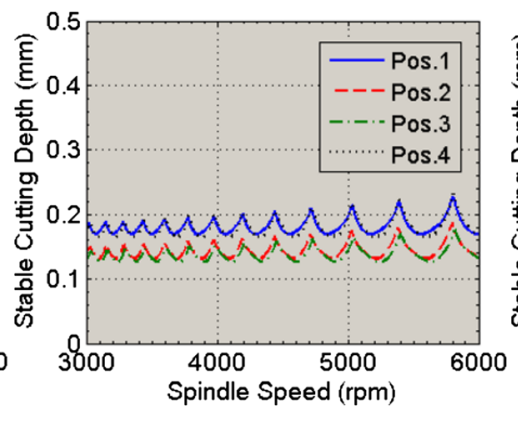

b

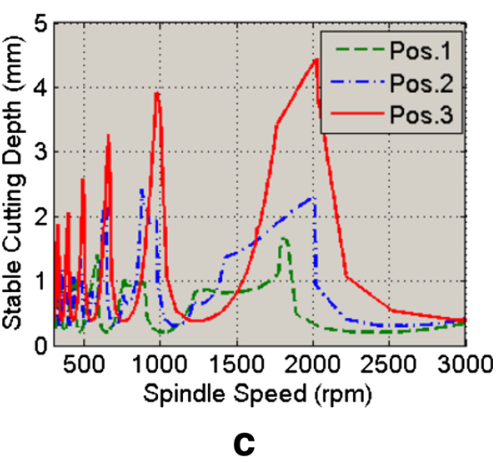

Fig. 4 Position-dependent stability limits in robotic milling (a) Tool 1 (b) Tool 2 (c) Tool 3

\subsubsection{Effect of cross transfer function}

In most of the stability solutions, the effect of cross transfer functions are ignored [13] i.e. In other words, Gxy and Gyx in Eq. (4) are taken to be zero. However, in robotic milling the flexible and complex kinematic chain may introduce cross transfer functions under certain conditions, which may affect stability diagrams once they are considered in Eq. (4). In this section, the conditions where the cross transfer functions may have significant effect on stability diagrams are discussed through simulations. The eigenvalue of the milling system given in Eq. (5) is solved numerically when cross transfer functions are considered in the solution. The direct and cross transfer functions measured at tool 1 are plotted in Fig. 6a, where it is seen that the amplitude of the cross transfer function is around $50 \%$ of Gxx and $25 \%$ of Gyy. The effect of the cross transfer function on milling stability is investigated for several cases as plotted in Fig. 6b to Fig. 6d. In case 1, down milling mode is simulated in feed $\mathrm{x}$ direction for quarter, half and full immersion. In case 2, the milling mode is altered to up milling whilst maintaining the same feed direction, where a quarter and a half immersion are considered. In case 3 , feed direction is altered to $y$ whilst keeping the other conditions the same with case 2 .

In Fig. 6b, it can be observed that cross transfer functions do not significantly affect stability limits. However, as the milling mode is altered to up milling, cross transfer function significantly and adversely affects milling stability especially for quarter immersion milling (see Fig. 6c). It is seen that the stability regions becomes narrower as cross transfer function
Fig. 5 Asymmetrical transfer function and its effect on stability diagrams (a) Comparison of FRF at the tip of Tool 1 when it is mounted to $\mathrm{CNC}$ and robotic milling setup (b) Effect of feed direction on the resultant FRF (c) Effect of feed direction on the stability diagrams for asymmetrical FRF

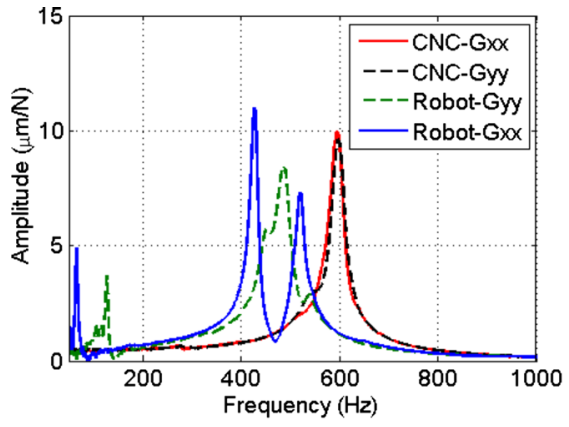

a

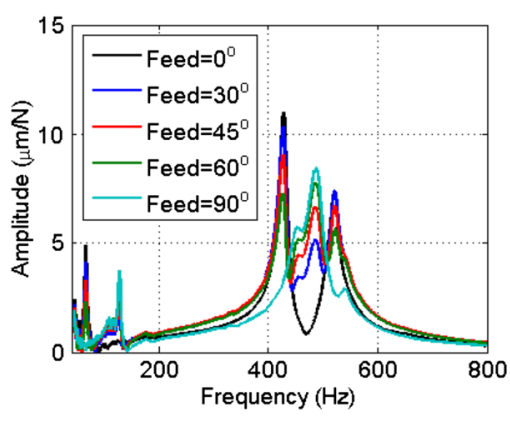

b

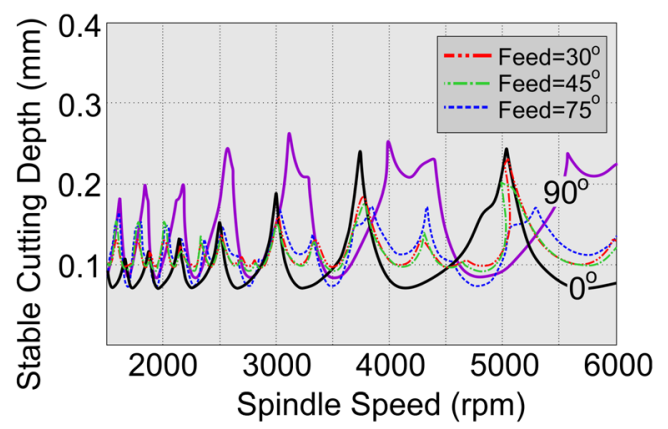

C 
Fig. 6 Effect of cross transfer function on stability diagrams for tool 1 (a) Direct and cross transfer functions (b) Down milling, feed -x (c) up milling, feed -x (d) Up milling, feed $-\mathrm{y}$

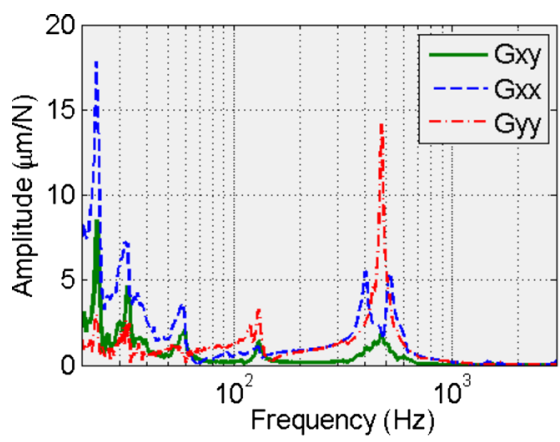

a

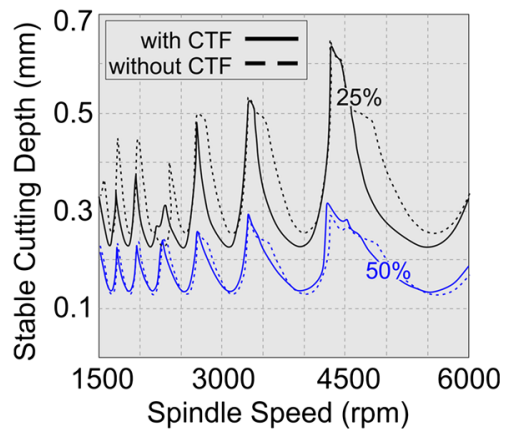

C
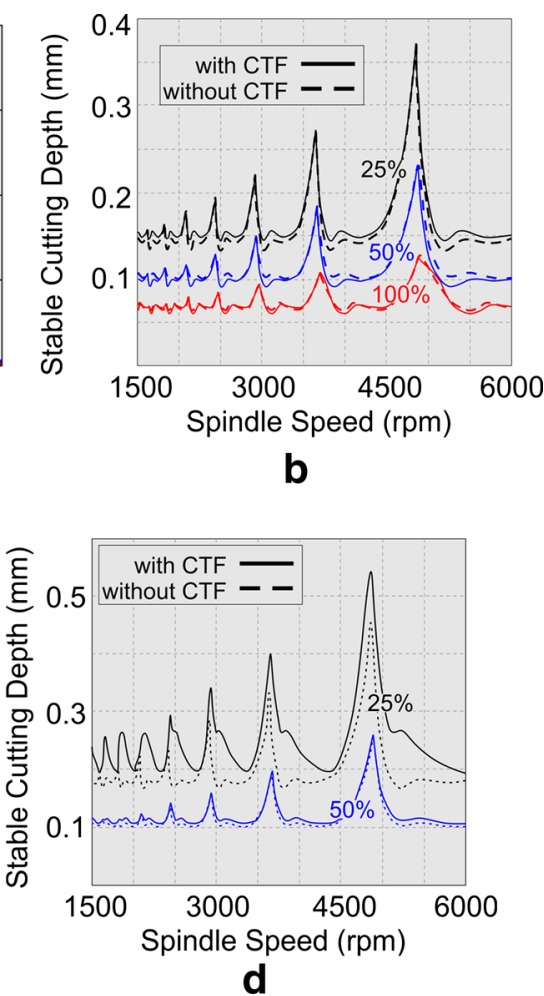

is included in the solution. The effect of cross transfer function, especially in quarter immersion milling, becomes favour of stability limits when the feed direction is altered to $y$ axis (see Fig. 6d). Through this example, it is shown that cross transfer function affects milling stability even if the amplitude of the cross transfer function is relatively small with respect to the direct transfer functions.

There are cases where milling stability is governed by the hexapod modes, as discussed before in this section. It was previously [15] identified that the cross transfer functions are even more significant at hexapod modes. This case is also illustrated through simulation of stability diagrams for full immersion face milling as plotted in Fig. 7. The measured tool point FRFs in Gxx, Gyy and Gxy are plotted in Fig. 7a, where it is seen that the dominant modes are less than $200 \mathrm{~Hz}$ and the cross transfer function is even more significant at all modes. The corresponding stability diagram for full immersion face milling is plotted in Fig. 7b. It is clearly seen that as the cross transfer function is considered in the solution, the stable cutting depth substantially change.

The flexible and complex kinematics in industrial robots introduce cross transfer functions even to the tool tip FRF. In this section, through simulations, it is shown that cross transfer function, Gxy, substantially affects stability diagrams once it is included into the solution. Having discussed several effects of robotic milling on process stability through simulations, the next section presents experimental results to support the evidences found out by simulations.
Fig. 7 Effect of cross transfer function on stability diagrams for tool 3 (a) Direct and cross transfer functions (b) Stability diagrams

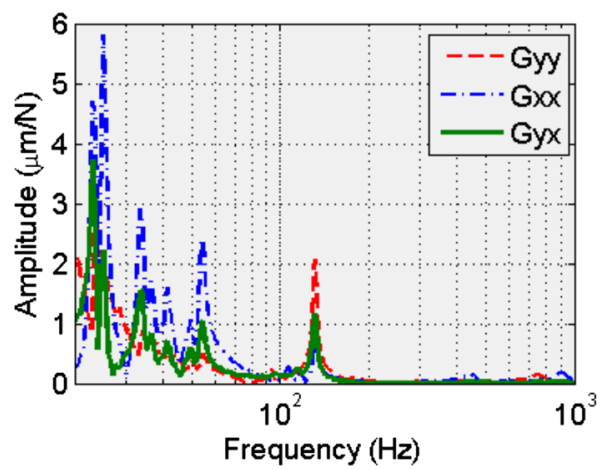

a

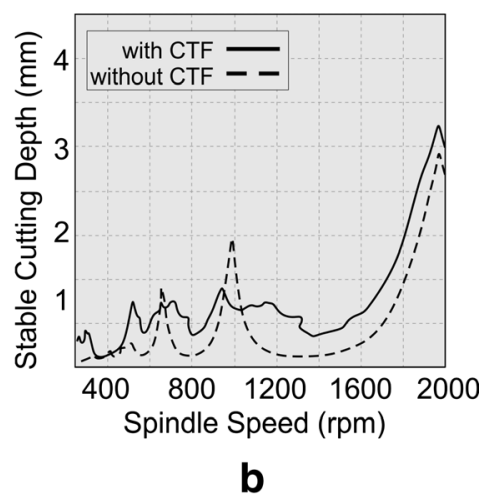




\subsection{Experimental results}

The effects of hexapod robot on milling stability is observed and verified through experimental results, where the test conditions are given in Table 3. The cutting tests are conducted on AL7075-T6, which is suitable for dry machining, where the cutting force coefficients are $\mathrm{Ktc}=800 \mathrm{MPa}, \mathrm{Krc}=300 \mathrm{MPa}$. Test 1 and test 2 are conducted to assess the effect of machining location and feed direction on stability. test 3 is done to see the effect of tooling on stability of robotic milling, where in test 4 the effect of cross transfer function is demonstrated. In the tests, vibration at the hexapod platform and the cutting sound is measured to assess stability.

The simulations discussed in the previous sections showed that cross transfer function does not significantly affect stability for cases over $40 \%$ radial immersion. Thus, in test 1 , test 2 and test 3 , the stability diagrams are simulated by CutPro $\odot$, where cross transfer function is ignored. In test 4 , the stability diagrams are simulated by introducing CTF to the stability solution [13] and eigenvalue is solved numerically. The simulated stability diagrams and experimental results are plotted in Fig. 8.

In test 1 , stability diagrams are calculated using the FRFs measured at randomly selected four machining locations, where the cutting tests are conducted at location 1 . In Fig. 8a, it is seen that the stability diagrams do not vary significantly with position when the feed is in the $x$ direction, which is altered to $y$ in test 2 . The stability diagram for Test 2 is plotted in Fig. 8b, where significant variation is observed in stability diagrams with respect to test 1 . The absolute stability limit and stability lobes shift with machining location and feed direction. Two completely different diagrams are obtained as the feed direction is altered from $x$ to $y$, due to the asymmetrical dynamic response at the tool tip.

The stability diagrams for Test 3 are calculated using the FRFs measured at four machining locations for feed direction $x$ and $y$ (see Fig. 8c). It is seen that the stability diagrams do not vary much even the feed direction is in $y$, contrary to Test 2 . Besides, the absolute stability limit is significantly higher than that of tool 1 . So, it can be said that milling with tool 2 in feed $y$ direction would lead to higher chatter-free cutting depth.

Table 3 Experimental cases

\begin{tabular}{lllll}
\hline Test & Tool no. & Radial depth & Cut type & Feed direction \\
\hline Test 1 & Tool 1 & Half & Down & $x$ \\
Test 2 & Tool 1 & Half & Down & $y$ \\
Test 3 & Tool 2 & Half & Down & $x$ \\
Test 4 & Tool 1 & Quarter & Up & $x$ \\
\hline
\end{tabular}

In test 4 , the stability diagrams are calculated with and without CTF. A significant difference is observed between the two cases, especially at the right hand side of the stability pockets, which is verified through representative cutting tests conducted at left, centre and right of a pocket around $4500 \mathrm{rpm}$. The observations of the effect of the hexapod robot on milling stability are done for the first in this paper. In this respect, it can be said that this paper contributes to the understanding of stability of robotic milling.

\section{Variable spindle speed strategy for improved stability}

As discussed through simulations and experimental results, the stability lobes may become position-dependent due to the varying dynamics of the hexapod robot. Hence, the spindle speed providing the same stable cutting depth shifts depending on the varying natural frequency. A similar case was studied in machining of turbine blades [16], where the dynamics of the workpiece vary as it is machined due to changing stiffness and mass as a result of material removal. It was shown that adjusting the spindle speed according to the varying dynamics may decrease process time about $40 \%$. In this paper, a similar strategy is applied in robotic milling of AISI $316 \mathrm{~L}$ steel. Tool 1 is used to introduce variable dynamics. The feed direction is selected as $x$ for minimum variation in the stability lobes. The radial depth of cut is selected as $60 \%$ through radial stability optimisation done using CutPro $\odot$. Stability lobes are generated for 20 machining locations (see Fig. 9a) within the area to be machined. The stability lobes are plotted as $3 \mathrm{D}$ and $2 \mathrm{D}$ to clearly visualise the variations in the stability limits, in Fig. 9b and Fig. 9c, respectively. The cutting speed is limited to $150 \mathrm{~m} / \mathrm{min}$ (1900 rpm) per tool manufacturer recommendation.

As the stability diagrams are observed, the maximum stable cutting depth is around $0.12 \mathrm{~mm}$ at $1875 \mathrm{rpm}$ (see Fig. 9c). However, it may be possible to achieve a higher stable cutting depth if the spindle speed is adjusted according to varying tool tip dynamics. Two tests are performed at $0.15 \mathrm{~mm}$ of cutting depth at constant and variable spindle speed. When constant spindle speed is applied, local chatter marks are observed throughout the machined surface. However, these chatter marks are eliminated by applying variable spindle speed and a better surface quality is achieved as shown in Fig. 9a, where the FFT of vibration in $x$ direction is also plotted. As a result, it is shown that productivity can be increased up to $25 \%$ if the spindle speed is adjusted according to the changing dynamics. 
Fig. 8 Stability tests (a) Test 1 (b) Test 2 (c) Test 3 (d) Test 4

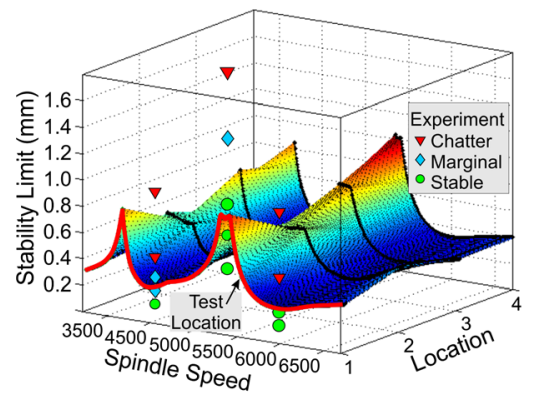

a

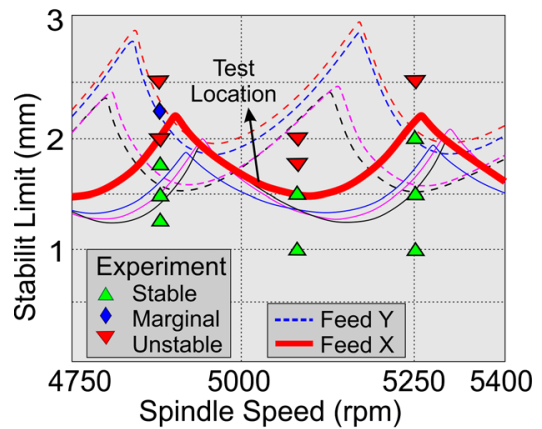

C

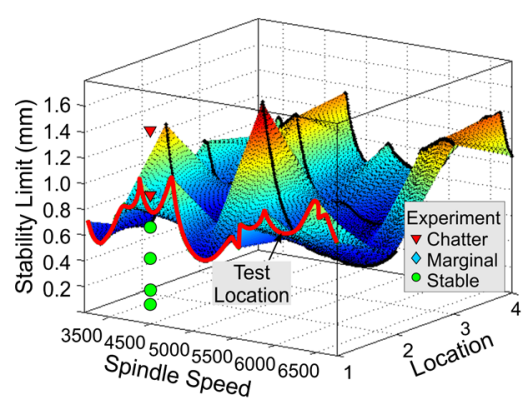

b

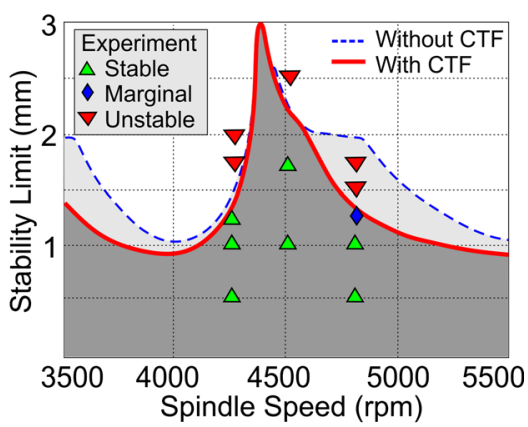

d

\section{Conclusions}

In this paper, the effects of a hexapod robot, utilised for mobile milling, on stability are discussed through simulations and experimental results. It is shown that the hexapod robot governs stability for face milling type of operations and it affects the tool tip dynamics by introducing asymmetrical flexibility. As a result, the stability diagrams depend on the feed direction. As the flexibility introduced by the robot is position- dependent, the stability diagrams may also become positiondependent at several cases. The experimental results verified such conclusions derived from the simulations. The conditions where the cross transfer functions may significantly affect stability diagrams are identified through simulations and verified by experiments. It is found out that when the radial depth of cut is less than $50 \%$ of the tool diameter the cross transfer function become significant for stability predictions. The position-dependent stability diagrams are dealt with
Fig. 9 Variable spindle speed strategy in robotic milling (a) Machined surface and sound FFTs (b) 3D Stability diagram (c) 2D Stability diagram
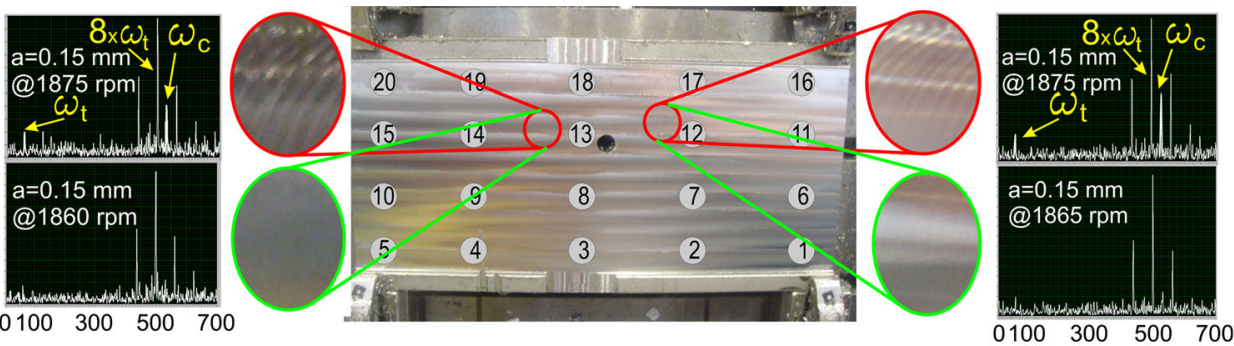

a

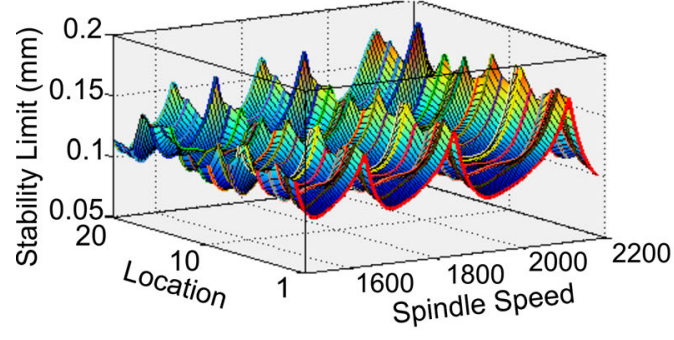

b

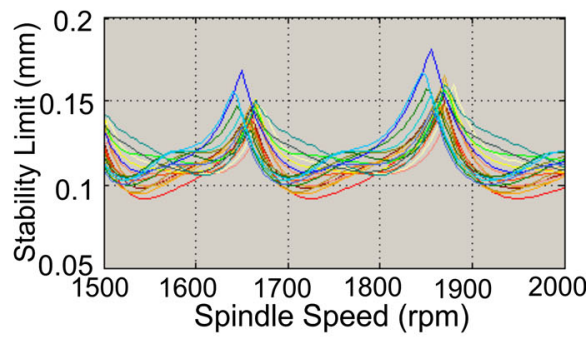

C 
variable spindle speed strategy. It is shown that by adjusting the spindle speed to the varying stability diagrams the stable cutting depth and hence the productivity can be improved up to $25 \%$. Considering these conclusions, this study contributes to the understanding of stability of robotic milling through clear identifications.

Acknowledgments The authors gratefully acknowledge the support of ESPRC, The University of Manchester and The University of Sheffield under NNUMAN Programme with grant number EP/J021172/1.

Open Access This article is distributed under the terms of the Creative Commons Attribution 4.0 International License (http:// creativecommons.org/licenses/by/4.0/), which permits unrestricted use, distribution, and reproduction in any medium, provided you give appropriate credit to the original author(s) and the source, provide a link to the Creative Commons license, and indicate if changes were made.

\section{References}

1. Uriarte L, Zatarain M, Axinte D, Yague-Fabra J, Ihlenfeldt S, Eguia J, Olarra A (2013) Machine tools for large parts. CIRP Ann 62(2): 731-750

2. Budak E (2006) Analytical models for high performance milling. Part II: process dynamics and stability. Int J Mach Tool Manuf 46: 1489-1499

3. Abele E, Weigold M, RothenBücher S (2007) Modelling and identification of an industrial robot for machining applications. CIRP Ann 56(1):387-390

4. Pan Z, Zhang H, Zhu Z, Wang J (2006) Chatter analysis of robotic machining process. J Mater Process Technol 173:301-309
5. Zaghbani I., Songmene V., Bonev I., 2013, An experimental study on the vibration response of a robotic machining system, Proceedings of the Institution of Mechanical Engineers, Part B: Journal of Engineering Manufacture, 227/6:866-880

6. Tlusty J, Ziegert J, Ridgeway S (1999) Fundamental comparison of the use of serial and parallel kinematics for machine tools. CIRP Ann 48(1):351-356

7. Rehsteiner F, Neugebauer R, Spiewak S, Wieland F (1999) Putting parallel kinematics machines (PKM) to productive work. CIRP Ann 48(1):345-350

8. Fassi I, Wiens GJ (2000) Multi axis machining: PKMs and traditional machining centers. J Manuf Process 2:1-14

9. Weck M, Staimer D (2002) Parallel kinematic machine tools - current state and future potentials. CIRP Ann 51(2): 671-683

10. Shneor Y, Portman VT (2010) Stiffness of 5-axis machines with serial, parallel and hybrid kinematics: evaluation and comparison. CIRP Ann 59(1):409-412

11. Law M, Ihlenfeldt S, Wabner M, Altintas Y, Neugebauer R (2013) Position-dependent dynamics and stability of serial-parallel kinematic machines. CIRP Ann 62(1):375-378

12. Axinte DA, Allen JM, Anderson R, Dane I, Uriarte L, Olarra A (2011) Free-leg hexapod: a novel approach of using parallel kinematic platforms for developing miniature machine tools for special purpose operations. CIRP Ann 60(1):395-398

13. Budak E, Altintas Y (1995) Analytical prediction of stability lobes in milling. CIRP Ann 44(1):357-362

14. www.fennetic.net

15. Tunc L.T., Shaw J., 2015, Experimental study on investigation of dynamics of hexapod robot for mobile machining, International Journal of Advanced Manufacturing Technology, http://dx.doi. org/10.1007/s00170-015-7600-6

16. Budak E, Tunc LT, Alan S, Ozguven HN (2012) Prediction of workpiece dynamics and its effects on chatter stability in milling. CIRP Ann 61(1):339-342 